continue to do so. For this expansion to be successful further investigation into its effectiveness is warranted.

1 Fonagy $\mathrm{P}$, Lemma A. Does psychoanalysis have a valuable place in modern mental health services? Yes. BMJ 2012; 344: e1211.

2 Bee P, Bower P, Lovell K, Gilbody S, Richards D, Gask, L, et al. Psychotherapy mediated by remote communication technologies: a meta-analytic review. BMC Psychiatry 2008; 8: 60 .

3 Fishkin R, Fishkin L, Leli U, Katz B, Snyder E. Psychodynamic treatment, training, and supervision using internet-based technologies. J Am Acad Psychoanal Dyn Psychiatry 2011; 39: 155-68.

Francesca L. Bell MRCPsych, CT3 in psychiatry, Camden Psychodynamic Psychotherapy Service, Camden and Islington NHS Foundation Trust, London, UK, email: franbell@doctors.org.uk

doi: $10.1192 / p b .37 .4 .144$

\section{Teaching undergraduate psychiatry in a forensic setting}

Typically, medical students do not learn about psychiatry in forensic settings. Depending on their interests and their medical school, they might have access to special study modules or elective placements in their final year. We introduced undergraduate psychiatry placements at our regional forensic service in New Zealand and surveyed the experiences and attitudes of our students over the past 2 years. The placement includes experience of medium-secure in-patients, community, prison and court liaison services.

McGauley \& Campbell ${ }^{1}$ asked whether medical students needed to know anything about forensic psychiatry and concluded that forensic psychiatry taught students about managing chronic illness, working with complex patients, understanding stigma and security, learning about personal and organisation dynamics and experiencing multidisciplinary, cross-disciplinary and multi-agency working. Such issues are relevant to all psychiatric specialties and some areas of medicine.

The University of Otago Medical School, Dunedin, was the first to be established in New Zealand. There are about 270 students each year. Students study psychological medicine throughout the 2nd year, and 8-week attachments, divided between two different areas of psychiatry, are compulsory in the 4th year. Students attend placements as part of the clinical team and complete logbooks of experiences, including team working and ethics. They see patients and learn about the assessment, formulation and management of common mental health problems. They regroup for weekly medical school teaching and undertake an examination with written and practical components.

We designed an online survey, which the medical school emailed to our 15 students who had spent their psychiatry placement with our forensic service; 73\% (11 students) responded. They were asked about the expectations and experiences they had had, the disorders they had learnt about, what they had enjoyed and what could have been improved.

Interestingly, 27\% were anxious about starting their placement. Most students thought that they would be learning mainly about 'legal issues' and seeing patients in prison. Some had 'no idea' what to expect. All respondents gained experience of seeing patients with schizophrenia, bipolar disorder, drug and alcohol problems and the problems associated with psychological trauma and head injury. Over
$70 \%$ also gained experience of seeing patients with personality disorders. Depressive and anxiety disorders were less commonly encountered, with around half of students gaining experience of these. We learnt that about $27 \%$ of respondents felt unsafe at some point during their placement and subsequently introduced a first-day security and safety induction and distributed a leaflet explaining the nature of forensic services and the placement, which we were told was useful

All students were positive and stated that they had enjoyed their placements. Over $70 \%$ rated the placement as 'excellent'. They appreciated seeing patients in a variety of settings which included in-patient, community, prison and court, and gained experience of a variety of mental health problems. Some students remarked that the placement was one of the best they had done in their undergraduate training to date. Forensic settings can therefore provide useful and enjoyable experiences to students as they learn about psychiatry as undergraduates.

1 McGauley G, Campbell C. Do medical students need to know anything about forensic psychiatry? Crim Behav Ment Health 2004; 14 (suppl 1): S6-11.

John P. Jacques, Consultant Forensic Psychiatrist and Senior Clinical Lecturer, Regional Forensic Service, Southern District Health Board, Dunedin, New Zealand, email: johnjacques77@doctors.org.uk

doi: $10.1192 / p b .37 .4 .145$

\section{Recruitment into psychiatry is working, but we are responsible for maintaining the momentum}

It is no secret that psychiatry has always struggled to recruit adequate numbers of doctors. This has led to the Royal College of Psychiatrists launching its Recruitment Strategy, implemented by a recruitment team. Their targets are to increase recruitment to core psychiatric training, achieving a 50\% increase in applications and a 95\% fill rate by the end of their 5 -year campaign. It is therefore most encouraging to learn that those foundation year (FY) doctors who are exposed to psychiatric placements are almost ten times more likely to embark on a career in the specialty. BMJ Careers reports ${ }^{1}$ that $15 \%$ of those FY2 doctors who undertook psychiatry placements applied for core psychiatry training, as compared with a mere $1.8 \%$ of those with no psychiatry exposure during their Foundation Programme. At last, a reason for quiet optimism, perhaps.

However, if we are to succeed in helping the College achieve its targets, it is clear that supervising clinicians have an important role to play. Archdall et al's qualitative study assessing medical students' perspectives of psychiatry postattachement, ${ }^{2}$ makes it patently clear that positive role models are a key factor in influencing eventual career choice. Respondents valued enthusiasm, eagerness to teach and motivation in those they were attached to as the most important qualities. It is highly likely that similar factors come into play with respect to influencing foundation doctors in choosing to pursue a career in psychiatry. Therefore, it is critical that those of us who are fortunate enough to supervise students and recently qualified doctors are fully conscious of our powers to positively influence recruitment. With great power comes great responsibility, as the saying goes, so the future of our specialty lies in our own hands. 